\title{
Densidad de ungulados en bosques de baja y alta presión de caza en el nororiente de la Amazonía peruana
}

\section{[Ungulates density in low and high hunting pressure forests in north eastern of Peruvian Amazonia]}

\author{
Rolando Aquino1, Luis López², Iris Arévalo³, Gabriel García², Elvis Charpentier² \\ ${ }^{1}$ Universidad Nacional Mayor de San Marcos, Facultad de Ciencias Biológicas, Lima, Perú \\ ¿Universidad Nacional de la Amazonía Peruana, Facultad de Ciencias Biológicas, Iquitos, Perú \\ 3Universidad Científica del Perú, Facultad de Ciencias e Ingeniería, Iquitos, Perú \\ *e-mail: raquinoy2005@yahoo.es
}

\begin{abstract}
Resumen
Se proporciona información sobre tamaño de rebaño en pecaríes y densidad poblacional de éstos y otros ungulados para los bosques de baja y alta presión de caza del nororiente peruano. Durante el recorrido de $610 \mathrm{~km}$ de transecto de noviembre-diciembre 2012 en bosques de baja presión de caza y de $1218 \mathrm{~km}$ desde febrero-junio y agosto-octubre 2013 en bosques de alta presión de caza, fueron registrados 76 y 40 encuentros pertenecientes a seis y cinco especies, respectivamente; siendo los más comunes Pecari tajacu (35\%) y Mazama americana (28\%). El tamaño de rebaño en $P$. tajacu varió desde 2 a 8 individuos (tamaño promedio: $3,4 \pm 1,6$ a $6 \pm 1,2$ ). La densidad poblacional estimada para los ungulados fue más alta en bosques de baja presión de caza sobresaliendo Tayassu pecari con 23,8 individuos $/ \mathrm{km}^{2}$, seguido por $P$. tajacu con 14,4 individuos $/ \mathrm{km}^{2}$, en tanto que la más baja fue para Tapirus terrestris $\left(0,08\right.$ individuos $\left./ \mathrm{km}^{2}\right)$ y correspondió a los bosques de alta presión de caza. En los bosques de baja presión de caza, los encuentros más frecuentes con ungulados ocurrieron en el palmal de altura (46\%) y palmal de planicie (31\%), en tanto que en el pantano arbóreo no hubo encuentros.
\end{abstract}

Palabras clave: Ungulados, tamaño rebaño, densidad poblacional, tipos de vegetación, frecuencia de encuentros.

\begin{abstract}
Information on peccary herd size and population density of these and other ungulates are provided for low and high hunting pressure forests in the northeastern Peruvian Amazonia. During the survey of $610 \mathrm{~km}$ transect from November-December 2012 in low hunting pressure forests and $1218 \mathrm{~km}$ from February to June and August to October 2013 in high hunting pressure forests were recorded 76 and 40 encounters from six and five species, respectively; being the most common Pecari tajacu (35\%) and Mazama americana (28\%). Herd size in P. tajacu ranged from $2-8$ individuals (mean size $3.4 \pm 1.6$ to $6 \pm 1.2$ ). Population density estimated to ungulates were higher in low hunting pressure forests and Tayassu pecari it excelled with 23.8 individuals $/ \mathrm{km}^{2}$, followed by $P$. tajacu with 14.4 individuals $/ \mathrm{km}^{2}$, while the lowest was to Tapirus terrestris (0.08individuals $/ \mathrm{km}^{2}$ ) and corresponded to the high hunting pressure forests. In the low hunting pressure forests, the more frequent encounters with ungulates occurred in the highland Palmal (46\%) and lowland Palmal (31\%), while in the swamp arboreal there were no encounters.
\end{abstract}

Key words: Ungulates, group size, population density, vegetation types, encounters frecuency. 


\section{INTRODUCCIÓN}

Entre los componentes de la fauna silvestre, los ungulados son los más apreciados para la caza y se debe al valor de su piel y carne (Bodmer et al., 1988) por lo que figuran entre los más requeridos para la venta en los mercados de las principales ciudades de la Amazonía peruana. Estos animales también cumplen funciones ecológicas en el bosque ya sea como dispersor o depredador de semillas de numerosas especies vegetales (Bodmer, 1991). A pesar de su importancia ecológica y económica, pocos son los estudios y la mayoría fueron llevados a cabo en la Reserva Comunal Tamshiyacu - Tahuayo y Yavarí-Mirí (Bodmer et al., 1996, 1997; Gottdenker,1996; Hurtado y Bodmer, 2004), Madre de Dios (Kirby y Padilla, 1998; Endo et al., 2010), Reserva Nacional Pacaya Samiria (Bodmer et al., 1999; Aquino et al., 2001), cuenca del río Pucacuro (Aquino et al., 1999), micro cuenca del río Alto Itaya (Aquino et al., 2007) y Alto Purús (Lleellish et al., 2007); para el resto prácticamente no existe información, mientras que en algunas cuencas la caza y la reducción de hábitats por efecto de la deforestación avanzan inconteniblemente. Por otro lado, los habitantes ribereños que son los principales beneficiarios tampoco valoran a estos animales, muy por el contrario se dedican a la destrucción de sus hábitats y a la caza desmedida, cuya consecuencia en el mediano plazo sería una drástica disminución de sus poblaciones, en particular de Tapirus terrestris, puesto que su baja tasa reproductiva no responde favorablemente a una alta presión de caza. Por el protagonismo en la economía de los habitantes ribereños y su importancia ecológica hemos considerado de interés contar con información sobre el estado actual de las poblaciones en bosques de baja y alta presión de caza, así como determinar los tipos de vegetación más frecuentados para sus hábitos alimenticios. Investigaciones cercanas al área de estudio fueron llevados a cabo en la Reserva Nacional Pucacuro (Álvarez, 1997; IIAP, 2001) y Lote 104 (ERM, 2007), los cuales estuvieron orientados al inventario, uso y conservación de la fauna silvestre en general, en tanto que en la micro cuenca del río Pucacuro fue específicamente sobre pecaríes (Aquino et al., 1999). En referencia a los bosques de alta presión de caza localizados entre las cuencas de los ríos Tigre-Nanay, los únicos estudios fueron conducidos en el sector del río Alto Itaya (Navarro y Terrones, 2006; Aquino et al., 2007) y tratan sobre la abundancia y presión de caza de mamíferos, entre ellos los ungulados; para el resto como los bosques aledaños a los ríos Alto Nanay y Huangana yacu afluente del río Tigre prácticamente no existe información. Para los bosques de baja presión de caza presentes en el río Curaray tampoco existe información acerca de los ungulados y la fauna silvestre en general, salvo los estudios específicos sobre primates. La carencia de información sobre ungulados para esta parte de la Amazonía peruana nos motivó a la conducción de este estudio, cuyos objetivos estuvieron orientados a determinar la abundancia y sus preferencias por los tipos de vegetación para sus actividades. El estudio se llevó a cabo de Noviembre a Diciembre del 2012 en bosques aledaños al río Curaray y de Febrero a Junio y Agosto a Octubre del 2013 entre las cuencas de los ríos Tigre-Nanay. Los resultados obtenidos se presentan en este reporte.

\section{MTERIALES Y MÉTODOS Área de estudio}

El estudio fue conducido en el nororiente de la Amazonía peruana y comprendieron los bosques de baja presión de caza del río Curaray muy cerca del límite con Ecuador y bosques de alta presión de caza entre las cuencas de los ríos Tigre y Nanay (Tabla 1, Figura 1 y 2). Ambos forman parte del denominado "Eco-región bosques húmedos del Napo" (Dinerstein et al., 1995), por lo que es considerado como una de las eco-regiones con mayor biodiversidad en el mundo. Con excepción del río Curaray, estos bosques por su cercanía a los centros poblados se encuentran muy alterados debido a la extracción de madera de valor comercial y de hojas de Lepidocaryum tenue ("irapay"), así como por la caza y cosecha de frutos silvestres, en particular de Mauritia flexuosa ("aguaje"), Oenocarpus bataua ("ungurahui") y Astrocaryum murumuru ("huicungo"). Tanto los bosques del río Curaray como de las cuencas Tigre-Nanay cuentan con ciertas particularidades tal como se describen a continuación: 
Río Curaray: correspondió a los bosques de baja presión de caza, cuyos lugares de censos fueron definidos próximos al límite fronterizo con Ecuador. Aquí predominaron los bosques de terraza alta, terraza media y terraza baja, incluyendo los aguajales mixtos. La vegetación en los bosques de colina baja y de terraza alta estuvo mayormente representada por árboles de porte alto, entre 25 a $30 \mathrm{~m}$, con algunos emergentes de hasta $40 \mathrm{~m}$. En los bosques de terraza media y terraza baja o inundable, la vegetación fue más bien de porte mediano, entre 20 a $25 \mathrm{~m}$ de altura, con algunos emergentes hasta $30 \mathrm{~m}$, muchos de ellos cubierto por enmarañados de bejucos y lianas. La fauna silvestre nos pareció relativamente abundante, en particular primates y pecaríes. Los censos fueron conducidos en ambas márgenes del río, desde las orillas hasta unos $5 \mathrm{~km}$ al interior del bosque. En estos bosques fueron diferenciados los siguientes tipos de vegetación:

Monte alto: conformado casi en un $100 \%$ por vegetación arbórea de porte alto, entre 20 a $25 \mathrm{~m}$ y algunos emergente arriba de los $30 \mathrm{~m}$. Sotobosque abierto y piso compacto. Entre las especies fueron comunes por su relativa abundancia Macrolobium angustifolium ("pashaco"), Eschweilera spp. ("machimango"), Couma macrocarpa ("leche huayo"), Chrysophyllum spp. ("caimitillo") Cedrelinga cateniformis ("tornillo"), Eschweilera spp. ("machimango"), Parahancornia sp. ("naranjo podrido"), Pouteria spp. ("caimitillo"), Hymenaea spp. ("azúcar huayo") y Vantanea spp. ("añuje rumo"). Entre las palmeras, las más frecuentes fueron $A$. murumuru e Iriartea sp. ("pona"). Este tipo de vegetación fue común en los bosques de colina baja y terraza alta.

Monte bajo: conformado por árboles de porte bajo entre 15 a $20 \mathrm{~m}$ de altura, la mayoría de ellos cubierto por enmarañados de bejucos y lianas. Sotobosque cerrado y poblado por plantas herbáceas como Calathea sp. ("bijao"), Bactris sp. ("ñejilla") y Costus sp. ("cañagria"). En este tipo se incluye la vegetación de ribera. Entre las especies fueron comunes Couropita guianensis ("ayahuma") Inga spp.("shimbillo"), Cecropia spp. ("cético"), Rheedia sp. ("charichuelo"), Annona sp. ("anona") y Passiflora sp. ("granadilla"). Este tipo de vegetación fue común en bosques de terraza media y terraza baja.

Palmal de altura: compuesto mayormente por palmeras entre 20 a $25 \mathrm{~m}$ de altura y entremezclada con árboles superiores a los 25 $\mathrm{m}$ de altura como $M$. angustifolium y Eschweilera spp.; sotobosque generalmente abierto y poblado algunas veces por palmeras de $L$. tenue y en otras por plantas herbáceas perennes. Entre las palmeras sobresalieron por su mayor frecuencia $O$. bataua, Socratea sp. ("huacrapona"), Iriartea sp., A. chambira, A. murumuru, Phytelephas macrocarpa ("yarina"), Scheelea cephalotes ("shapaja") y Scheelea sp. ("shebón"). Este tipo de vegetación estuvo presente mayormente en bosques de colina baja y terraza alta.

Palmal de planicie: compuesto en más del $70 \%$ por Mauritia flexuosa ("aguaje"), asociada con Mauritiella sp. ("aguajillo"), Euterpe sp. ("chonta") y algunas especies arbóreas como Ficus spp. ("renaco"). Este tipo de vegetación estuvo presente en bosques de terraza media y terraza baja, cuyo piso con abundantes raíces zancos estaba cubierto por agua negra. Los aguajales mixtos se caracterizaron por su disposición en fajas de hasta $1 \mathrm{~km}$ de largo y entre 50 a $200 \mathrm{~m}$ de ancho.

Varillal: compuesto por árboles y arbustos tupidos entre 10 a $25 \mathrm{~m}$ de altura y algunos emergentes arriba de $30 \mathrm{~m}$; la mayoría de fuste recto y esclerófilos, similar a los varillales de arena blanca, excepto por los suelos que fueron de tipo areno arcilloso y eventualmente arenoso. Entre las especies emergentes sobresalieron Parkia sp., Manilkara sp. yEschweilera sp. Este tipo de vegetación estuvo presente únicamente al Sur del río Curaray y en "manchales" en la cima de los bosques de Colina baja y en terraza alta.

Pantano arbóreo: compuesto por árboles de mediana altura (20 a 25m); sotobosque abierto y piso cubierto con agua y abundantes raíces zancos. Vegetación conformado en un alto porcentaje por Ficus spp. y entremezclado con Tachigalia sp. ("tangarana"), Guarea sp. ("requia") y escasas palmeras, entre ellas Euterpe sp. Este tipo de vegetación fue común en bosques de terraza baja. 
Es oportuno mencionar que la diferenciación de los tipos de vegetación tuvo como propósito comparar las preferencias de los ungulados por estos hábitats durante sus diversas actividades.

Cuencas de los ríos Tigre-Nanay: correspondió a los bosques de alta presión de caza y estuvieron conformados por los ríos Huanganayacu, Alto Itaya y Alto Nanay. En Huangana yacu predominaron los bosques de terraza media y terraza baja incluyendo los aguajales mixtos, en tanto que en los ríos Alto Itaya y Alto Nanay los bosques de colina baja, en cuya cima la vegetación arbórea fue de tipo "varillal", es decir, árboles de fuste recto y delgado, con alturas entre 15 a $25 \mathrm{~m}$ y algunos emergentes arriba de $30 \mathrm{~m}$; sotobosque semi cerrado y poblado mayormente por $L$. tenue, piso compacto de tipo areno arcilloso y cubierto por un colchón de hojarascas. A diferencia de las cimas, los árboles en las laderas se caracterizaron por ser de porte alto (por encima de $25 \mathrm{~m}$ ), fuste grueso y copas amplias. En general, los bosques mostraron serias alteraciones por la extracción de madera de valor comercial y de hojas de $L$. tenue, así como por la caza y cosecha de frutos, por lo que fue común la presencia de trochas y campamentos en uso. La fauna silvestre, en particular los denominados de tamaño grande como ungulados nos pareció relativamente escaso. En cada uno de estos lugares los censos fueron conducidos en ambas márgenes del río, desde la orilla hasta $5 \mathrm{~km}$ al interior del bosque.

\section{Generación de información en campo}

Para el registro de ungulados fueron abiertos ocho transectos en el río Curaray y 12 entre las cuencas Tigre-Nanay; es decir, cuatro por cada lugar de censo, cuyas longitudes en ambos casos variaron de 3 a $5 \mathrm{~km}$. En los censos participaron dos grupos de observadores conformado por un profesional y un asistente de campo. Los censos fueron de ida y vuelta, desde las 06:30 a 11:30 h (ida) y desde 14:00 a 16:30 h (vuelta) y consistió en el recorrido por los transectos a una velocidad promedio de $1.0 \mathrm{~km} / \mathrm{hora}$ con paradas cada cierto trecho por uno a dos minutos para detectar cualquier movimiento o ruido. En el Curaray, los censos también fueron realizados en horario nocturno desde las 18:30 $\mathrm{h}$ hasta las 22:30 $\mathrm{h}$ y estuvieron orientados al registro de Mazama gouazoubira ("venado colorado"), M. americana ("venado colorado") y Tapirus terrestris ("sachavaca"). Es oportuno precisar que estos ungulados son de hábitos diurnos y nocturnos. Cada vez que hubo avistamiento se procedió a anotar la hora, especie, número de individuos (en el caso de pecaríes y cuando fue posible el conteo), distancia perpendicular desde el primer individuo observado al transecto, tipo de vegetación dominante y actividad al momento de la observación. De un total de $1648 \mathrm{~km}$ de censos diurnos, $1218 \mathrm{~km}$ correspondió a los bosques de alta perturbación y el resto a los de ligera perturbación al cual se adicionan $180 \mathrm{~km}$ de censos nocturnos (Tabla 2).

\section{Análisis de datos}

El bajo número de individuos y/o rebaños registrados para los ungulados no permitió calcular la densidad (D) mediante la aplicación del software Distance, por lo que se usó la fórmula $\mathrm{D}=\mathrm{N} / 2 \mathrm{dL}$ (Burnham et al., 1980); donde $\mathrm{N}$ es el número de grupos $\mathrm{y} / \mathrm{o}$ individuos registrados, $\mathrm{L}$ es la longitud $(\mathrm{km})$ recorrida y $\mathrm{d}$ es la distancia perpendicular promedio del primer animal observado al transecto, la misma que fue distinta para cada especie. En pecaríes, el cálculo de individuos $/ \mathrm{km}^{2}$ se obtuvo multiplicando $D$ por el tamaño promedio del rebaño. En el caso del río Curaray, la longitud (L) fue de $610 \mathrm{~km}$ para el cálculo de densidad de $M$. americana, M. gouazoubira y $T$. terrestris y $430 \mathrm{~km}$ para pecaríes.

Para comparar la diversidad de ungulados usamos el conteo de rebaños e individuos por tipos de vegetación como los datos crudos y luego aplicamos el Índice de equidad de Shannon-Wiener y de dominancia de Simpson (Moreno, 2001).

\section{RESULTADOS}

Durante los recorridos fueron registrados 116 encuentros pertenecientes a cinco especies; de ellas, 76 correspondió al río Curaray y 40 a los bosques entre las cuencas Tigre-Nanay (Tabla 3). Los encuentros más comunes en Curaray y Tigre-Nanay fueron con Pecari tajacu ("sajino") y M. americana que en conjunto representaron el $63 \%$ y la menos común $T$. pecari ("huangana") con escasos cinco registros equivalente al $4 \%$. 
Entre los ungulados, los pecaríes son los únicos que viven en rebaños. El rango de variación en el tamaño y tamaño promedio para $P$. tajacu fue determinado de rebaños donde fue posible el conteo completo y varió de 5 a 8 individuos en el río Curaray (tamaño promedio: $6 \pm 1,2, N=6$ ) y de 2 a 7 entre las cuencas Tigre-Nanay (tamaño promedio: $3,4 \pm 1,6, N=16)$. Para $T$. pecari no fue posible un conteo completo porque los rebaños al momento de contactarlos se encontraban muy dispersos, excepto uno que fue hallado en plena locomoción en bosque de colina baja conformado por aproximadamente 85 individuos incluyendo los infantes.

La densidad poblacional más alta para las cinco especies correspondió al Curaray, sobresaliendo entre ellas $T$. pecari con 23,8 individuos $/ \mathrm{km}^{2}$ y la más baja fueron para $T$. terrestris y M. gouazoubira, ambas entre las cuencas Tigre-Nanay con apenas 0,08 y 0,2 individuos $/ \mathrm{km}^{2}$, respectivamente (Tabla 4). En los bosque de alta presión de caza, los dos únicos ejemplares de $T$. terrestris fueron observados en Huangana yacu, en el resto solamente huellas, los mismos que fueron muy escasas; en tanto que $T$. pecari no fue observada en ninguno de los lugares de censos y la única vez que registramos huellas fue en Huangana yacu, lo que indicaría que ambas especies se encuentran en proceso de extinción local.

En el río Curaray, de los seis tipos de vegetación diferenciados, en cinco fueron observados a los ungulados. Los encuentros más frecuentes ocurrieron en palmal de altura y palmal de planicie que en conjunto sumaron $77 \%$ de los avistamientos; no obstante, los valores obtenidos para los índices de Shannon - Wiener $(H=1,5)$ y Simpson $(D=0,76)$ indican que la diversidad de especies fue más alta en el palmal de altura, confirmando así como el hábitat más frecuentado por los ungulados en bosques del río Curaray. $P$. tajacu, $M$. gouazoubira y $M$. americana fueron observados con más frecuencia en palmal de altura y $T$. pecari y $T$. terrestris en palmal de planicie (Tabla 5). Por otro lado, el varillal fue la menos frecuentada por los ungulados con apenas $22 \%$ de los registros pertenecientes a dos especies, entre ellas M. gouazoubira, en tanto que en el pantano arbóreo no hubieron registro.

\section{DISCUSIÓN}

Entre los ungulados, la densidad poblacional estimada para los pecaríes en bosques de baja presión de caza fue ampliamente superior a los estimados para los bosques de alta presión de caza y mayor a los reportados para los sectores de moderada y persistente caza de la Reserva Nacional Pacaya Samiria (Bodmer et al., 1997; Aquino et al., 2001), micro cuenca del río Pucacuro (Aquino et al., 1999), Yavarí-Mirí (Bodmer et al., 1997), Madre de Dios (Kirby y Padilla (1998) y Alto Purús (LLeellish et al., 2007), excepto al de $T$. pecari para el área sin caza del Parque Nacional Manú (Endo et al., 2010) que fue más alta a nuestro resultado. En nuestro caso, la densidad para $T$. pecari fue estimada sobre la base de 85 individuos porque fue el único rebaño donde el conteo fue cercano al $100 \%$. Por el tamaño se habría tratado de un sub grupo, en tanto que los otros rebaños observados probablemente superaban los 100 individuos. La densidad estimada para $M$. gouazoubira, M. americana y $T$. terrestris en bosques de ligera presión de caza también fue mayor a las reportadas para el Pacaya Samiria (Bodmer et al., 1997; Aquino et al., 2001) y Yavarí-Mirí (Bodmer et al., 1997), excepto el de $T$. terrestris, que resultó ligeramente superior a nuestro resultado. Lo contrario ocurrió en los bosques de alta presión de caza donde con excepción de $P$. tajacu, la densidad para el resto de ungulados estuvo muy por debajo a los reportados para las áreas antes mencionadas, por lo que en estos bosques ya habría sobre caza. La baja densidad, en particular de $T$. terrestris y nulo contacto con T. pecari, estaría relacionada no solamente con la caza, sino también con la deforestación. En efecto, durante nuestra permanencia en Huangana yacu, Alto Itaya y alto Nanay encontramos grupos de personas dedicados a la extracción ilegal de madera con fines comerciales, quienes contaban con cazadores para el aprovisionamiento de "carne de monte". Otro factor para la escasez y ausencia de ungulados en bosques de alta presión de caza también sería la escasez de frutos, en particular de M. flexuosa, 0 . bataua y Euterpe sp. que constituyen importantes recursos alimenticios para los ungulados, pero que a su vez tienen gran aceptación por los citadinos, por lo que para la cosecha las plantas son derribadas, tal como constatamos 
en los tres sectores donde fue evidente la escasez de estas especies. Lo contrario ocurrió en los bosques de baja presión de caza donde los ungulados y demás componentes de la fauna silvestre todavía fueron abundantes, porque en este sector la caza y extracción de madera es muy esporádica por su lejanía de los centros poblados y porque además la producción de frutos es alta durante todo el año, tal es así que con frecuencia fueron observados infantes en cualquier época, no solamente de ungulados sino también de otros mamíferos.

El alto porcentaje de encuentros con ungulados en palmal de altura y palmal de planicie indica que en selva baja los bosques primarios con abundantes palmeras son hábitats de mucha importancia para estos animales. En el caso de pecaríes, la preferencia por estos tipos de vegetación estaría relacionada con la abundancia de frutos que son consumidos en grandes cantidades por estos animales. Por otro lado, la mayor frecuencia de encuentros de $P$. tajacu, M. gouazoubira y M. americana en palmal de altura y de $T$. pecari y $T$. terrestris en palmal de planicie es un claro indicador de que el primer grupo tiene preferencia por los bosques de tierra firme donde se alimentan principalmente de frutos de palmeras (Bodmer, 1989), en particular de O. bataua, Iriartea sp. y $A$. murumuru y el segundo grupo por los bosques inundables a los cuales se han adaptado (Bodmer, 1990) y donde los frutos de M. flexuosa y Mauritiella sp. figuran entre sus principales recursos alimenticios. En referencia al varillal, la escasa presencia de ungulados y de otros mamíferos estaría en estrecha relación con la baja producción de frutos, el mismo que estaría relacionado con la composición florística que es relativamente pobre en cuanto a diversidad tal como indica el resultado obtenido por Oñate Calvín (2012) para el varillal cercano a la ciudad de Iquitos con apenas 114 especies. Finalmente, la ausencia de encuentros con ungulados en el pantano arbóreo de hecho tiene relación con la escasez de frutos de palmeras, puesto que en este tipo de vegetación predominan las especies de la familia Moraceae, entre ellas Ficus spp. Brosimun sp, y Pouroma sp. , cuyos frutos al parecer no son muy requeridos por los ungulados, en particular por T. pecari, al menos así lo indican los análisis del contenido estomacal (Bodmer, 1989).

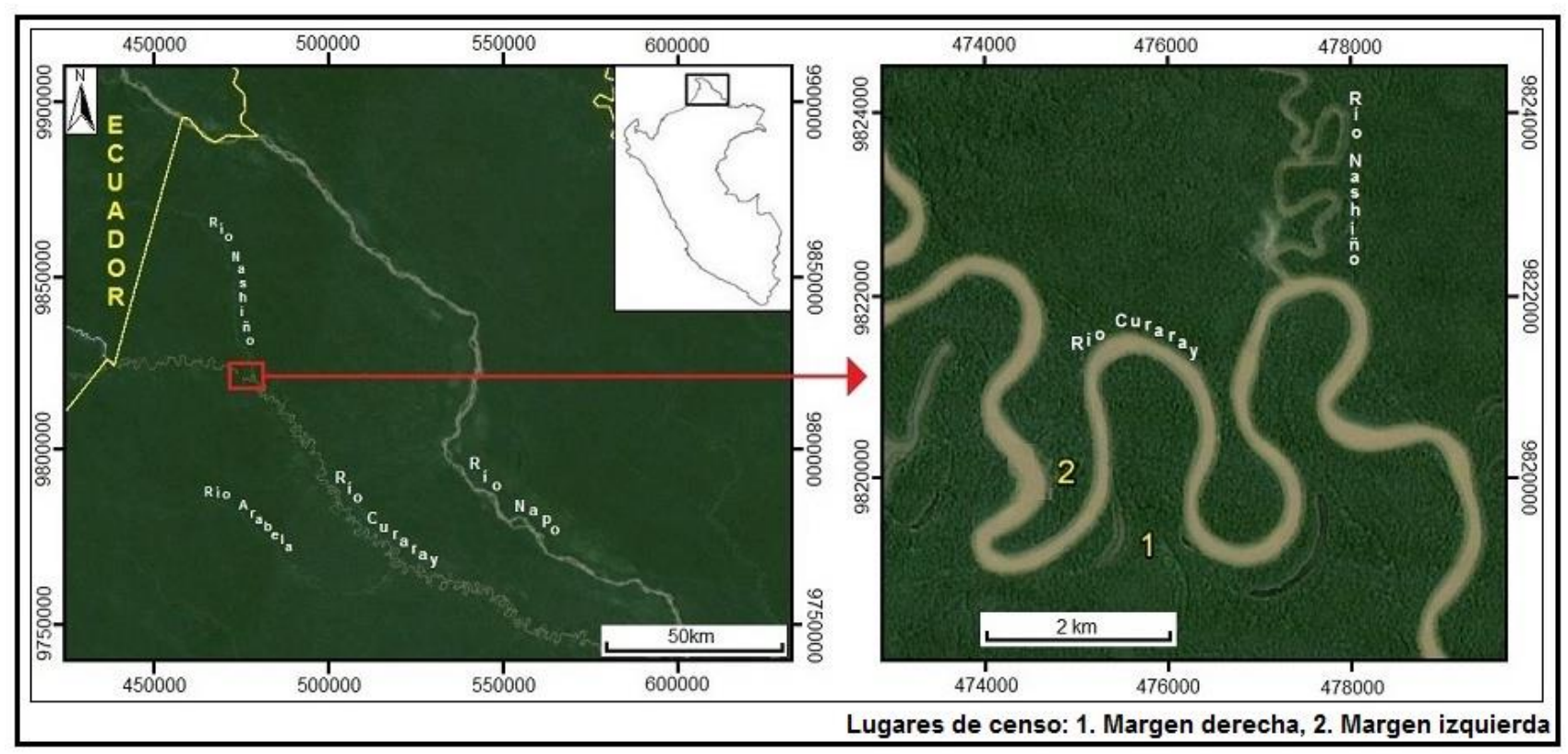

Figura 1. Mapa mostrando los lugares de censos en el río Curaray 


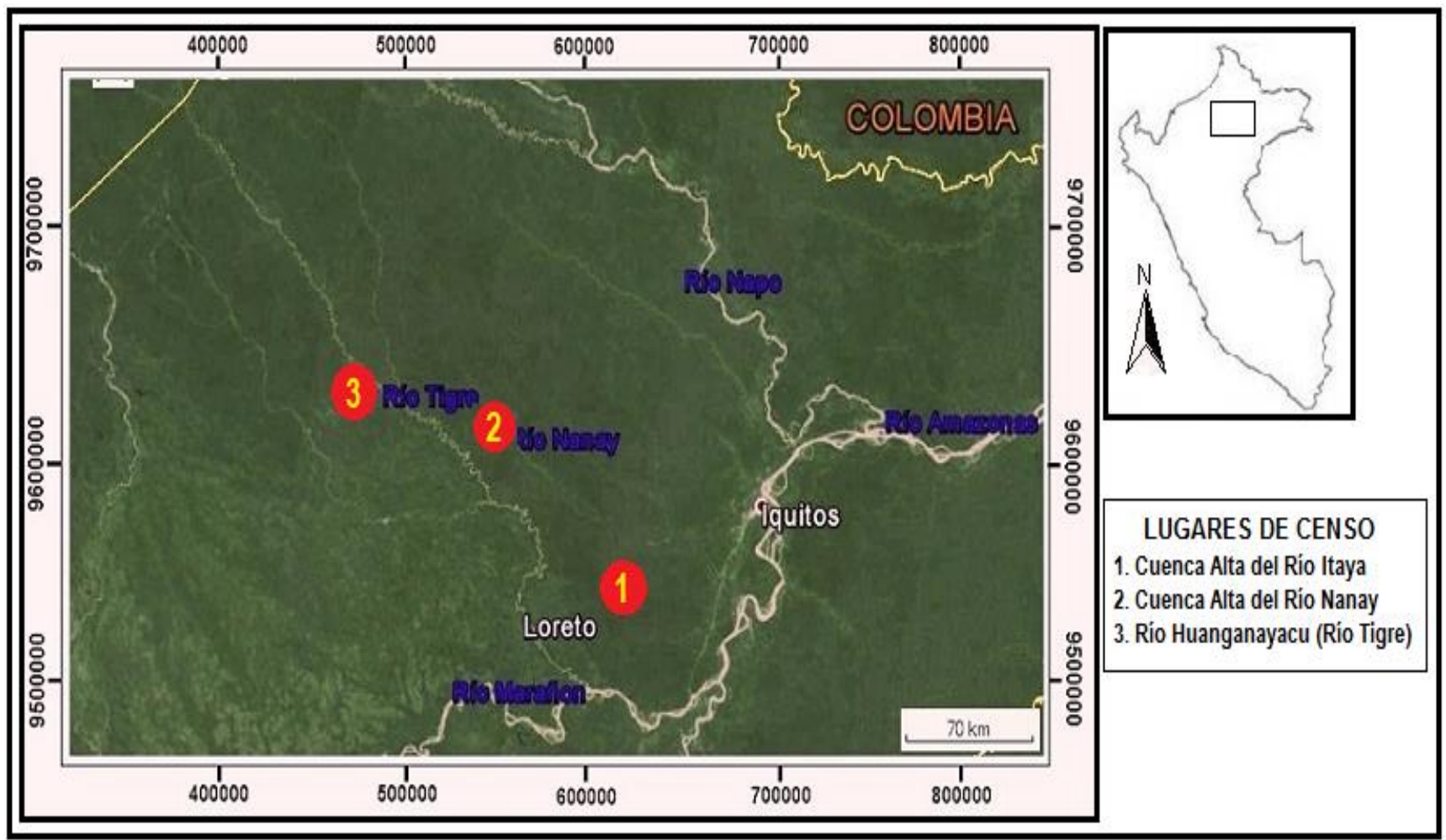

Figura 2. Mapa mostrando los lugares de censos entre las cuencas de los ríos Tigre - Nanay

Tabla 1. Lugares de censos en las áreas de muestreo del nororiente peruano

\begin{tabular}{llcc}
\hline \multicolumn{1}{c}{ Áreas de muestreo } & \multicolumn{1}{c}{$\begin{array}{c}\text { Lugares de } \\
\text { censos* }\end{array}$} & Coordenadas (UTM) E/N & $\begin{array}{c}\text { Tipos de bosques } \\
\text { predominantes }\end{array}$ \\
\hline Curaray & Margen derecha (1) & $475731 / 9821084$ & $\mathrm{Cb}, \mathrm{Ta}, \mathrm{Tm}$ \\
$\begin{array}{l}\text { (Bosques baja presión } \\
\text { de caza) }\end{array}$ & Margen izquierda (2) & $474749 / 9821868$ & $\mathrm{Ta}, \mathrm{Tm}, \mathrm{Tb}$ \\
$\begin{array}{l}\text { Tigre-Nanay (Bosques } \\
\text { alta presión de caza) }\end{array}$ & $\begin{array}{l}\text { Alto Itaya (1) } \\
\text { Huangana yacu (2) } \\
\text { Alto Nanay (3) }\end{array}$ & $615679 / 9540649$ & $\mathrm{Cb}, \mathrm{Ta}$ \\
\end{tabular}

Cb: Colina baja, Ta: Terraza alta, Tm: Terraza media, Tb: Terraza baja y Ag: Aguajal mixto.

* Los números en paréntesis corresponden a los lugares de censos en las Figs. 1 y 2.

Tabla 2. Longitud recorrida durante los censos por transecto

\begin{tabular}{lcc}
\hline \multirow{2}{*}{ Horario de censo } & \multicolumn{2}{c}{ Áreas de muestreo } \\
& Curaray & Tigre-Nanay \\
\hline Diurno & 430 & 1218 \\
Nocturno & 180 & - \\
Total & 610 & 1218 \\
\hline
\end{tabular}


Tabla 3. Rebaños y/o individuos de ungulados registrados en las áreas de muestreo del nororiente peruano.

\begin{tabular}{lcccc}
\hline Especies & Curaray & Tigre-Nanay & Total & $\%$ \\
\hline Pecari tajacu & 19 & 22 & 41 & 35 \\
Tayassu pecari & 5 & - & 5 & 4 \\
Mazama gouazoubira & 15 & 4 & 19 & 16,5 \\
Mazama americana & 20 & 12 & 32 & 28 \\
Tapirus terrestris & 17 & 2 & 19 & 16,5 \\
Total & 76 & 40 & 116 & 100 \\
\hline
\end{tabular}

Tabla 4. Densidad poblacional de ungulados en las áreas de muestreo y comparación con otras áreas.

\begin{tabular}{|c|c|c|c|c|c|c|}
\hline \multirow{2}{*}{ Áreas de estudio } & \multicolumn{5}{|c|}{ Densidad poblacional (Indiv./(km²) } & \multirow{2}{*}{ Fuente } \\
\hline & $\mathrm{Pt}$ & $\mathrm{Tp}$ & $\mathrm{Mg}$ & $\mathrm{Ma}$ & $\mathrm{Tt}$ & \\
\hline Yavarí-Mirí & 2,1 & 9,7 & & 0,96 & 1,7 & Bodmer et al., 1997 \\
\hline Tahuayo-Blanco & 1,4 & 6,6 & & & & Bodmer et al., 1997 \\
\hline Pucacuro & 1,52 & 3,9 & & & & Aquino et al., 1999 \\
\hline Samiria & 0,6 & 4,5 & & 1,3 & 0,3 & Bodmer et al., 1997 \\
\hline $\begin{array}{l}\text { Samiria } \\
\text { Madre de Dios }\end{array}$ & $\begin{array}{l}2,4 \\
1,7\end{array}$ & $\begin{array}{c}10,5 \\
7,2\end{array}$ & & 0,7 & 0,1 & $\begin{array}{l}\text { Aquino et al., } 2001 \\
\text { Kirby y Padilla, } 1998\end{array}$ \\
\hline Alto Purús & 2,5 & 9,2 & & & & Lleellish et al., 2007 \\
\hline PN Manú (con caza) & 7,8 & 5,8 & & & & Endo et al.,2009 \\
\hline PN Manú (sin caza) & 8,03 & 43,8 & & & & Endo et al.,2009 \\
\hline Curaray & 14,4 & 23,8 & 1,4 & 2,7 & 1,5 & Este estudio \\
\hline Tigre-Nanay & 6,1 & & 0,27 & 0,49 & 0,08 & Este estudio \\
\hline
\end{tabular}

Pt: Pecari tajacu; Tp: Tayassu pecari; Ma: Mazama americana,Tt: Tapirus terrestris.

Tabla 5. Proporción (\%) y número de registros $(\mathrm{N})$ de ungulados de acuerdo a los tipos de vegetación en el río Curaray.

\begin{tabular}{|c|c|c|c|c|c|c|}
\hline \multirow{2}{*}{ Tipos de vegetación } & \multicolumn{5}{|c|}{$\begin{array}{l}\text { Especies (en orden de incremento de } \\
\text { masa corporal) }\end{array}$} & \multirow{2}{*}{ Tota } \\
\hline & P.t. & T.p. & M.g & M.a. & T.t. & \\
\hline \multicolumn{7}{|l|}{ Monte alto } \\
\hline$\%$ & 5 & & 27 & 30 & 6 & 16 \\
\hline $\mathrm{N}$ & 1 & & 4 & 6 & 1 & 12 \\
\hline \multicolumn{7}{|l|}{ Monte bajo } \\
\hline$\%$ & & & & 10 & & 3 \\
\hline $\mathrm{N}$ & & & & 2 & & 2 \\
\hline \multicolumn{7}{|l|}{ Palmal de altura } \\
\hline$\%$ & 53 & 40 & 60 & 45 & 29 & 46 \\
\hline $\mathrm{N}$ & 10 & 2 & 9 & 9 & 5 & 35 \\
\hline \multicolumn{7}{|l|}{ Palmal de planicie } \\
\hline$\%$ & 37 & 60 & & 15 & 65 & 31 \\
\hline $\mathrm{N}$ & 7 & 3 & & 3 & 11 & 24 \\
\hline \multicolumn{7}{|l|}{ Varillal } \\
\hline$\%$ & 5 & & 13 & & & 4 \\
\hline $\mathrm{N}$ & 1 & & 2 & & & 3 \\
\hline \multicolumn{7}{|l|}{ Total } \\
\hline$\%$ & 100 & 100 & 100 & 100 & 100 & 100 \\
\hline $\mathrm{N}$ & 19 & 5 & 15 & 20 & 17 & 76 \\
\hline
\end{tabular}




\section{AGRADECIMIENTOS}

Nuestro agradecimiento a The Mohamed bin Zayed Species Conservation Fund quien financió las expediciones para la evaluación de Atélidos y de otros primates en el nororiente de la Amazonía peruana, del cual aprovechamos para obtener información adicional acerca de los ungulados. Nuestro reconocimiento a Idea Wild por la donación de algunos materiales de campo que fueron de mucha utilidad. Hacemos extensivo nuestra gratitud a los asistentes de campo, en particular a Gilmer Montero de quien no nos cansaremos de elogiarlo por su espíritu alentador y de lucha por lograr nuestro propósito.

\section{REFERENCIAS BIBLIOGRÁFICAS}

Álvarez JA. 1997. Estado actual de la fauna silvestre en la propuesta Reserva Comunal del Pucacuro. En: Manejo de fauna silvestre en la Amazonía. T. Fang, R. E. Bodmer, R. Aquino y M. Valqui (eds), pp. 93-103. La Paz, Bolivia.

Aquino R., Bodmer RE, Pezo E. 1999. Evaluación de poblaciones del pecarí de collar ( Tayassu tajacu) y pecarí labiado ( $T$. pecari) en la cuenca del río Pucacuro, río Alto Tigre. En: Manejo y Conservación de Fauna Silvestre en América Latina. T. Fang, O. Montenegro y R. Bodmer (eds.), pp. 471- 480. La Paz, Bolivia.

Aquino R, Bodmer RE, Gil G. 2001. Mamíferos de la cuenca del río Samiria: Ecología Poblacional y Sustentabilidad de la Caza. Imprenta Rosegraff. Lima, Perú.

Aquino R, Terrones C, Navarro R, Terrones W. 2007. Evaluación del impacto de la caza en mamíferos de la cuenca del río Alto Itaya, Amazonía peruana. Rev Per Biol 14 (2), $181-186$.

Bodmer, RE, Fang TG, Moya L. 1988. Estudio y manejo de los pecaríes (Tayassu tajacu y $T$. pecari) en la Amazonía peruana. Matero 2: 18 - 25.

Bodmer RE. 1989. Frugivory in Amazonian Artiodactyla: Evidence for the evolution of the ruminant stomach. J. Zool. 222: $121-128$.

Bodmer ER. 1990. Responses of ungulates to seasonal inundations in the Amazon floodplain. J Trop Ecol 6, 191 - 201.
Bodmer, RE. 1991. Strategies of seed dispersal and seed predation in Amazonian ungulates. Biotropica 23, $255-261$.

Bodmer RE, Sowls L, Taber A. 1996. Importancia económica y utilización humana de los pecaríes. En: Pecaríes. W.L.R. Oliver (ed.), pp. 39 - 49. IUCN, Quito, Ecuador.

Bodmer RE, Aquino R, Puertas P, Reyes C, Fang T, Gottdenker N. 1997. Manejo y Uso Sustentable de Pecaríes en la Amazonia Peruana. Occassional Paper No. 18, Comisión de Supervivencia de Especies, IUCN, Quito, Ecuador.

Bodmer R, Allen C, Penn J, Aquino R, Reyes C. 1999. Evaluación del uso sostenible de la fauna silvestre en la Reserva Nacional Pacaya Samiria. Documento de Trabajo América Verde No. 4b. The Nature Conservancy.

Burnham KP, Anderson DL, Laake JL. 1980. Estimation of density from line transects sampling of biological populations. Wild Monogr 72, 1-202.

Dinerstein E., Olsen DM, Graham DJ, Webster $A L$, Prim SA, Book-binder MP, Ledec J. 1995. A conservation assessment of the terrestrial ecoregions of Latin America and the Caribbean. World Bank, WWF. Washingto D.C., USA.

Endo W, Peres CA, Salas E, Mori S, SanchezVega JL, Shepard GH, Pacheco V, Yu DW. 2010. Game vertebrate densities in hunted and nonhunted forest sites in Manu National Park, Peru. Biotropica 42(2), $251-261$.

ERM. 2007. Estudio de impacto ambiental y social de la prospección sísmica 2D de $445 \mathrm{~km}$ en el lote 104. Capítulo 3. Línea base ambiental, Lima, Perú.

Gottdenker NL. 1996. Reproductive ecology and harvest evaluation of peccaries in the Norheastern Peruvian Amazon. MSc. Thesis, University of Florida.

Hurtado J, Bodmer RE. 2004. Assessing the sustainability of brocket deer hunting in the Tamshiyacu Tahuayo Communal Reserve, northeastern Peru. Biological Conservation 116, 1- 7. 
IIAP 2001. Conservación y manejo de la biodiversidad de la cuenca del Pucacuro. Programa del aprovechamiento sostenible de la biodiversidad. Informe Técnico.

Kirby CA, Padilla P. 1998. An evaluation of the population and management of Tayassu tajacu and T. pecari in Madre de Dios, Perú. INRENA-CITES, Lima.

Lleellish M, Amanzo J, Hooker Y, Yalle S. 2007. Evaluación poblacional de pecaríes en el Alto Purús - Ucayali. Serie de publicaciones de flora y fauna silvestre. Instituto Nacional de Recursos Naturales, Lima, Perú. [En línea] $<$ http://www.inrena.gob.pe/iffs/iffs_bio div_estud_flora_fauna_silvestre.htm> [Consulta: 13 Febrero 2014].
Moreno, CE. 2001. Métodos para medir la biodiversidad. Manuales \& Tesis SEA, Vol. 1, Zaragoza, España.

Navarro R, Terrones C. 2006. Evaluación del impacto de la caza en mamíferos de la cuenca alta del río Itaya, Loreto. Tesis título Biólogo, Universidad Nacional de la Amazonía Peruana. Iquitos, Perú.

Oñate Calvín R. 2012. Caracterización y aprovechamiento de los varillales amazónicos. Contribución a la economía de las localidades. Tesis doctoral, Universidad Politécnica de Madrid, España. 